\title{
Una aproximación al panorama actual de las nuevas formas de consumo de drogas
}

\section{New forms of drug use: An overview}

\author{
Manuel Isorna Folgar*, Francisco Arias Horcajadas**. \\ * Universidad de Vigo. Facultad Ciencias Educación y Trabajo social. Grupo PsiConBi, Ourense, España. \\ ** Programa de Alcohol y Patología Dual. Hospital Doce de Octubre, Madrid, España.
}

$\mathrm{E}$ 1 consumo de drogas está muy arraigado en la "cultura" occidental y su consumo se relaciona con tradiciones, celebraciones o espacio de ocio. Sin embargo, estos consumos no están exentos de riesgos que dependen de la cantidad, frecuencia y patrón de consumo, así como de las características de la persona consumidora como edad, sexo y algunas condiciones de salud. Mientras la evidencia científica acumula pruebas de la toxicidad de las drogas, sigue existiendo una creciente oferta y demanda de las mismas debido principalmente a la aparición o el redescubrimiento de nuevas sustancias psicoactivas y "el atractivo" de las nuevas formas o patrones de consumo. Paradójicamente, pese a la creciente preocupación que suscitan estos nuevos patrones de consumo son muy pocos los trabajos realizados en nuestro país que analizan esta temática.

Si bien sabemos que la adolescencia suele ser un momento de búsqueda y experimentación y que muchos jóvenes prueban sustancias sin que eso implique necesariamente que tengan un problema de adicción, la evidencia disponible apunta a la aparición de conductas adictivas a edades cada vez más tempranas (Rial, Golpe, Barreiro, Gómez e Isorna, 2020). La última Encuesta Estatal sobre uso de Drogas en Enseñanza Secundaria (ESTUDES, 2021), señala una discreta disminución en el consumo de todas las sustancias psicoactivas en general. Sin embargo, la población juvenil española consume más alcohol y cannabis que la media eu- ropea (ESPAD, 2019) y otras formas de consumo como el cigarrillo electrónico $(\mathrm{CE})$ continúa ganando adeptos. Por lo tanto, nuestro objetivo es revisar estas nuevas formas y patrones de consumo y sus posibles implicaciones sociosanitarias, fundamentalmente para los más jóvenes.

\section{ALCOHOL}

Según la encuesta ESTUDES (Plan Nacional sobre Drogas, 2021) 311.200 estudiantes (chicos: 152.500, chicas: 158.000) han comenzado a consumir alcohol por primera vez durante el último año, número ligeramente inferior al obtenido en las dos ediciones anteriores. Además, aproximadamente 6 de cada 10 estudiantes ha bebido alcohol en los últimos 30 días, observando que el $40 \%$ del alumnado comenta haberse emborrachado en los 12 últimos meses, mientras que el 23,2\% lo ha hecho en el último mes, siendo las chicas quienes reconocen haberse emborrachado en mayor medida que los chicos y el 27,9\% ha realizado "binge drinking”, es decir, ha tomado 5 o más bebidas alcohólicas en un intervalo aproximado de dos horas. Este fenómeno se ha visto agravado en los últimos años debido a la proliferación de macrofestivales (aproximadamente 900 al año en España), los cuales están financiados en su mayor parte por la industria alcoholera (Torres, 2020). Es importante destacar que antes, durante y después de los mismos se consumen 
grandes cantidades de alcohol, tanto es que por este motivo en muchos festivales suelen utilizar diferentes métodos para impedir el consumo de alcohol por parte de los menores; un ejemplo de este método es la utilización de pulseras para diferenciar las personas mayores de edad de las que son menores (Adán, 2018).

Además del consumo en atracón o "binge drinking" también han surgido nuevas formas de consumo principalmente tratando de reducir el precio de la borrachera y, también, el tiempo en alcanzarla; la mayor parte son importadas del Reino Unido y EE. UU., en las que se utilizan otras superficies mucosas del organismo diferentes a la vía digestiva. Destacamos entre ellas:

1. El eyeballing: consiste en la aplicación directa de alcohol sobre la mucosa ocular. El efecto alcohólico conseguido probablemente sea bajo, pero generalmente se practica tras una ingesta importante de alcohol, por lo que los usuarios hablan de un mayor «subidón». Esta práctica presenta un elevado riesgo de lesiones corneales graves con eventual evolución a ceguera (Bersani et al., 2015).

2. Los oxy-shots o "chupitos de la risa": se consume el alcohol en dispositivos de nebulización junto con oxígeno, al igual que en los tratamientos broncodilatadores. Ello permite una mayor superficie de absorción y rapidez de acción, al obviar el filtro hepático. Con esta práctica podrían aparecer o agravarse patologías pulmonares. En 2011, la Dirección General de Salud Pública y Consumo del Govern Balear inmovilizó estos dispensadores de chupitos de alcohol inhalado, esgrimiendo el posible riesgo que acarrean para la salud (Saenz, 2011).

3. El tampodka, también conocido como «tampax on the rocks»: consiste en la aplicación en la vagina o el ano de tampones impregnados previamente en alcohol, generalmente vodka. Ello produce una absorción muy rápida y evita, inicialmente, el fetor enólico, por lo que parece ser popular en adolescentes para sortear el control paterno. Esta práctica provoca un aumento de la incidencia de lesiones e infecciones en las mucosas. A pesar de que es considerada como una leyenda urbana (Caudevilla, 2015), en España hay registrados casos (Fonseca, 2013).

4. Otra práctica frecuente en jóvenes es la mezcla de alcohol con bebidas energizantes (de alto contenido en cafeína, taurina y guaraná). Esta mezcla puede llevar a consumir más cantidad de alcohol por la falsa sensación de "control", acometer riesgos en la conducción y a medio plazo favorecer la dependencia del alcohol (Arria et al., 2011). Los consumidores subestiman el estado de intoxicación, por lo que suelen quedarse hasta más tarde, incrementando el consumo y participando en conductas de riesgo (Burillo-Putze, Hernández y Echeverría, 2012; Oteri, Salvo, Caputi y Calapai, 2007). El consumo de este tipo de bebidas energéticas puede provocar ansiedad, nerviosismo, insomnio, palpitaciones, e incluso, fibrilación auricular, convulsiones y miocarditis (Baez-Ferrer et al., 2020; Izquierdo et al., 2012). Por estos motivos, la Food and Drug Administration ha iniciado el proceso para ilegalizar las bebidas alcohólicas con cafeína existentes en ese país, como la popular Four loko, que toma su nombre de los 4 tipos de estimulantes que usa: cafeína, taurina, guaraná y ajenjo (absenta) y que posee una graduación alcohólica del 12\% (O’Brien, McCoy, Rhodes, Wagoner y Wolfson, 2008; Rehm, Shield, Joharchi y Schuper, 2012). En España, desde el año 2014, ha tenido mucho éxito entre los jóvenes el Jägerbomb, una mezcla que hace furor entre los jóvenes españoles y triunfa en los botellones y que une el licor alemán Jägermeister con un 35\% de graduación alcohólica y una bebida energética (principalmente Red Bull). El cóctel que llegó a España como "el chupitazo de moda" lleva tiempo triunfando en las redes sociales y se va amplificando su fama.

Otra forma de consumo por destacar son las gominolas alcohólicas u ositos borrachos, básicamente consiste en verter la bebida alcohólica en un recipiente lleno de ositos de gominola (u otro tipo de gominola de similares características), taparlo con papel film e introducirlo en la nevera durante 24 horas. Las gominolas absorberán el líquido y estarán listos para comer (aproximadamente 17 gominolas equivalen a una copa). Ha sido tal el éxito de esta fórmula que se ha industrializado su venta y distribución (https:/ / ositosconalcohol.com/).

Otra sustancia incorporada en ambientes de ocio por jóvenes que buscan el "colocón" es la bebida púrpura, (también denominadas "learn", "sizzurp”, "jarabe púrpura"), término que hace referencia a una mezcla de "jarabe para la tos" (hidrocloruro de prometazina) mezclado con soda (habitualmente Sprite) y/o alcohol, además de los caramelos Jolly Ranchers para darle sabor (Agnich, Stogner, Miller y Marcum, 2013; Elwood, 2001; Hart, Agnich, Stogner y Miller, 2014; Miuli et al., 2020). En ocasiones, los usuarios sin acceso al jarabe de prometazina, utilizan otro jarabe para la tos que contiene codeína (Chiappini, Schifano, Corkery y Guirguis, 2021). La Agencia Española del Medicamento y Productos Sanitarios (2016) recomienda especial atención por el uso de dextrometorfano por sus efectos adversos y los posibles efectos alucinógenos con dosis elevadas (Lessenger y Feinberg, 2008). El abuso de prometazina se ha relacionado con la sordera (Blakley y Schilling, 2008), e, incluso, con la muerte (Chiappini, Schifano, Corkery y Guirguis, 2021; Hart et al., 2014).

Posiblemente, con el objetivo de atraer a los jóvenes, en la última década, la industria ha creado una serie de bebidas denominadas "alcopops", "bebidas de diseño" o "bebidas alcohólicas saborizadas" (BAS), también conocidas como bebidas listas para tomar (RTD, del inglés Ready to Drink). 
A nivel mundial, las BAS más populares son productos como el Bacardi Breezer, el Smirnoff Ice, Mike's Hard Lemonade, Ron Cacique Mojito y Two Dogs (Buglass, 2011; Manzoni, 2014). Lo que realmente las define no es tanto su contenido alcohólico (que puede oscilar desde el 3,5\% al 20\%) sino la innovación, el diseño y marketing asociado, así como el estilo de presentación (envase). El uso de caracteres que sugieren los dibujos animados, tiras cómicas, "signos" juveniles, atractivas sugerencias sexuales, envases especiales que no se pueden apoyar (similar a un "test-tubo" de laboratorio) y que tienen que mantenerse en la mano, sabores dulces buscando los gustos infantiles y enmascarando el sabor del alcohol, colores estridentes y, a veces, luminosos, en definitiva, todos aquellos elementos que hacen que este tipo de bebidas sean muy atractivas para los jóvenes (Buglass, 2011). Hace años, Pascual (2002) ya señalaba la familiarización de los jóvenes con este tipo de bebidas, sobre todo en jóvenes de 14-15 años, con especial incidencia entre las chicas.

A los patrones de consumo por atracón o "binge drinking”, se ha unido otro patrón del consumo de alcohol preocupante, principalmente en las mujeres jóvenes, es la drunkorexia o ebriorexia, término utilizado por primera vez en el "The New York Times" (Kershaw, 2008) para nombrar la conducta de jóvenes que restringen el consumo de alimentos con alto aporte calórico para ingerir bebidas alcohólicas en exceso. Las personas que manifiestan estas conductas generalmente tienen conocimientos acerca del contenido energético de las bebidas alcohólicas y tratan de equilibrar la ingesta de alimentos para evitar incrementar su peso corporal. Desafortunadamente el mantenimiento de este "equilibrio" los lleva a dejar de comer drásticamente para poder consumir alcohol en forma desmedida (Chambers, 2008). El patrón habitual de la drunkorexia incluye contar las calorías de los alimentos y bebidas que ingerirán, dejar de comer por horas o días enteros previo a la ingestión de las bebidas alcohólicas y, después, incrementar la actividad física para quemar las calorías consumidas en exceso (Martínez, López-Espinoza, Navarro Meza, López-Uriarte y Salazar Estrada, 2014). Parece más común entre las mujeres universitarias entre 18 y 24 años con distorsión de la imagen corporal (Pietrabissa et al., 2018). La drunkorexia puede presentar consecuencias físicas y psicológicas graves (Villarino, 2012).

\section{TABACO}

Según el ESTUDES (2021), a lo largo del año 2021 empezaron a fumar en España algo más de 169.600 estudiantes de enseñanzas secundarias, siendo mayor el número de chicas (chicos: 74.500; chicas: 95.100). El tabaco es la segunda droga más extendida entre los estudiantes de 14 a 18 años tras el alcohol. En cuanto a las formas de consumo, el 49,2\% han declarado consumir en los últimos 30 días tanto cigarrillos de cajetilla como tabaco de liar o "roll-your-own" (RYO), siendo ya un $22,5 \%$ los que sólo utilizan RYO, casi duplicando lo registrado en 2019; un 14,1\%. La creencia por parte de los fumadores de que el RYO tiene menos riesgo para la salud, que contienen menos aditivos, que son menos perjudiciales o el ritual que supone su preparación son los principales motivos de este ascenso (Brown et al., 2015). Sin embargo, algunos estudios han encontrado mayores concentraciones de nicotina, alquitrán y monóxido de carbono (Laugesen, Epton, Frampton, Glover y Lea, 2009). Además, los fumadores de RYO muestran un mayor riesgo de cáncer de boca, laringe, faringe y pulmón que los fumadores de cigarrillos convencionales (Rolke, Bakke y Gallefoss, 2009; Young et al., 2012). La falsa creencia de que el RYO es menos dañino para la salud es mayor en jóvenes, sin embargo, los contenidos de nicotina, alquitrán y monóxido de carbono alcanzan valores de hasta el $70 \%$, el $85 \%$ y el $84 \%$, respectivamente, más de lo permitido para los cigarrillos convencionales (Calduch, Jiménez, San Segundo, Valle y Carlos-Roca, 2012). Más nicotina significa mayor poder de adicción; más alquitrán y monóxido de carbono se traduce en mayor capacidad de producir enfermedad. Las investigaciones también han demostrado que los consumidores de RYO tienden a ser más adictos (Joseph et al., 2018) y más jóvenes que los fumadores de cigarrillos tradicionales y pertenecen a grupos sociales de bajos ingresos (Young et al., 2012).

Al mismo tiempo, en los últimos años se va camino de normativizar el vapeo a través del cigarrillo electrónico (CE), como un sustituto o complemento del cigarrillo tradicional. Si bien se presentó como un medio seguro y aparentemente eficaz para lograr el cese del consumo de cigarrillo convencional, se ha convertido en lo que han denominado un caballo de Troya, ya que mediante su uso muchos adolescentes están expuestos a consumos de nicotina similares e incluso superiores a los cigarrillos convencionales (Jackler y Ramamurthi, 2019). Así en España, según recoge el ESTUDES (2021), el 44,3\% del alumnado entre 14 y 18 años ha utilizado en alguna ocasión el CE. El consumo en varones ha sido del 46,9\% y en mujeres del 41,7\%. Es de reseñar que de cada 10 estudiantes que han fumado tabaco en alguna ocasión, ocho de ellos han utilizado CE. Pero lo más destacable es que de cada 10 estudiantes que nunca han fumado tabaco, tres de ellos han utilizado CE en alguna ocasión. Entre aquellos que han fumado CE, únicamente un $8,5 \%$ lo ha hecho para reducir su consumo de tabaco o para dejar de fumar (porcentaje ligeramente superior en los chicos 9,3\% frente a $7,6 \%$ de las chicas), aunque su eficacia para dejar de fumar está siendo muy cuestionada (Córdoba, 2014; El Dib et al., 2017; Signes-Costa et al., 2019). Estos dispositivos producen un aerosol a partir del calentamiento de líquidos que contienen solventes (glicerina, propilenglicol), uno o más saborizantes, nicotina y, en ocasiones, derivados del cannabis (principalmente THC y CBD), por lo que se han vendido como una forma alternativa de obtener el efecto 
de la nicotina y THC sin estar expuesto a los efectos deletéreos de los demás componentes de un cigarrillo o "porro" convencional (Budney, Sargent y Lee, 2015; Monraz-Pérez, Regalado-Pineda y Pérez-Padilla, 2015). Por este motivo, actualmente se tiene una falsa noción de seguridad que ha favorecido la aceptación del consumo del CE y una gran confianza al utilizarlo, incluido el consumo de los derivados del cannabis, así según el ESTUDES (2021) el 5,3\% (6,7\% chicos; $3,7 \%$ chicas) de los adolescentes que han consumido cannabis en los últimos 30 días lo han fumado con este mecanismo. Sin embargo, los CE emiten carbonilos volátiles y metales (níquel, plomo, cromo) muchos de los cuales son tóxicos para el pulmón (Gotts, Jordt, McConnell y Tarran, 2019). En España, con un crecimiento medio del $25 \%$ anual, el número de vapeadores se sitúa por encima de las 562.500 personas en 2018 y el sector factura ya más de 88 millones de euros en nuestro país (Upev, 2019), destacando en ventas los JUULpods. En EEUU el uso de este CE se considera una "epidemia entre los jóvenes". Se presenta con un diseño de USB (se carga de hecho conectándose por USB a un ordenador o un enchufe), al que se insertan cápsulas con líquidos de sabores muy atractivos para los jóvenes (mango, menta, crème brulée, Virginia -sabor tabaco original-, pepino...). Ese líquido contiene diferentes químicos, pero, la gran diferencia con respecto a los demás cigarrillos electrónicos es que utiliza sales de nicotina y vapearse una cápsula entera (los JuulPods) equivale a fumarse unos 20 cigarrillos.

\section{CANNABIS}

Según el ESTUDES (2021), se estima que en el año 2018 han empezado a consumir cannabis 155.800 estudiantes siendo ligeramente mayor el número de chicas 83.200 que de chicos 72.600. La edad de inicio del consumo de esta droga comienza antes de los 15 años. Es importante destacar que el daño causado es inversamente proporcional a la edad de inicio, de manera que es cuatro veces mayor si se inicia a los 15 años (actual edad media de inicio en el consumo en España) que, si se hace a los 26 años; por ejemplo, se estima que hasta el $8 \%$ de la incidencia de esquizofrenia en la población adulta fumadora podría estar relacionada con el consumo de cannabis en jóvenes (Di Forti et al., 2019; Marconi, Di Forti, Lewis, Murray y Vassos, 2016). Incluso se ha demostrado que el consumo de cannabis de manera únicamente puntual puede producir cambios estructurales y cognitivos en el cerebro de los adolescentes (Orr et al., 2019). También se asocia con un aumento del riesgo de padecer trastornos del comportamiento y psicosis, este riesgo aumenta cuanta mayor frecuencia de consumo y mayor potencia del cannabis utilizado (Di Forti et al., 2019). Sin lugar a duda, la evidencia científica sobre los riesgos y consecuencias orgánicas, psíquicas y sociales asociadas a su consumo son cada vez más robustas (Rial et al., 2018; Volkow et al., 2016; WHO, 2016).
En cuanto a las nuevas formas de consumo del cannabis añadidas a las tradicionales como la fumada y vaporizada, destacan el hotboxing (el submarino) que consiste en que varios consumidores inhalan el humo o el aerosol de la marihuana, del hachís o del Butane Hash Oil (BHO) en un espacio cerrado y pequeño, entre los que se incluyen automóviles, cascos de moto gigantes, despensas, cabinas telefónicas, tiendas de campaña o armarios. Puede ser peligroso por el acumulo de $\mathrm{CO} 2$ y el incremento de otras toxinas y la transmisión de otras enfermedades (Oeltmann et al., 2006). Otra práctica en aumento es el "Shotgunning" que se refiere a inhalar humo de drogas ilícitas y luego exhalarlo directamente en la boca de otro (Perlman et al., 1997). Se relaciona con mayor gravedad de la adicción y más conductas de riesgo, existiendo escasa conciencia entre estos consumidores del riesgo de transmisión de enfermedades vía respiratoria (Welsh et al., 2012). Tanto el Hotboxing como el Shotgunning son formas de consumo social y se ha asociado a la transmisión de enfermedades infecciosas como la tuberculosis (French et al., 2019; Oeltmann et al., 2006).

Cocinar/Hornear (galletas, pasteles, brownies) es el método más popular después de fumar entre los jóvenes, así el 1,4\% han ingerido THC a través de alguno de estos productos en los últimos 30 días $(2 \%$ chicos y $0,7 \%$ de las chicas). Debido a que la absorción es más lenta, el inicio de los efectos se retrasa (con una concentración plasmática máxima media de 1 a 2 horas después de la ingestión, en contraste con los 5 a 10 minutos para las concentraciones plasmáticas máximas si se fuma), pero la duración de la intoxicación es más larga (Hazekamp, Ware, Muller-Vahl, Abrams y Grotenhermen, 2013). Debido a los efectos retardados de los comestibles, es posible que se consuman varias porciones seguidas antes de experimentar el "subidón" de la porción inicial. El consumo de una gran dosis de THC puede dar lugar a una mayor concentración del mismo, a una mayor intoxicación y un mayor riesgo de efectos adversos (Hancock-Allen, Barker, VanDyke y Holmes, 2015).

Por otra parte, el dabbing es una forma de consumir un concentrado de cannabis en forma de aceite, también denominados "budder", "Dab" "shatter" o "BHO (Butane Hash Oil)". Su extracción implica llevar adelante un proceso muy peligroso y complejo que involucra químicos altamente inflamables como el gas butano o dióxido de carbono. Como resultado obtiene un aceite pegajoso o "bad" que puede alcanzar hasta un $80 \%$ de THC en comparación con un $5-20 \%$ en los derivados de los cannabis tradicionales (Stogner y Miller, 2015). El "dab" se añade al dispositivo de dabbing, se calienta y, posteriormente, se nebuliza y se convierte en un aerosol que se inhala profundamente en una sola bocanada y se mantiene en los pulmones durante varios segundos (Anderson y Zechar, 2019; Raber, Elzinga y Kaplan, 2015). Debido a su alta concentración de THC, 
los riesgos de dependencia e intoxicación son muy altos (Stephens, Patel, Angelo y Frunzi, 2020).

La vía de administración del cannabis debe ser tenida en cuenta por que los efectos psicotrópicos ocurren dentro de los minutos posteriores a la inhalación por vía fumada y perduran de 2 a 4 horas, mientras que los efectos psicotrópicos del consumo oral generalmente ocurren dentro de los 30 a 60 minutos y duran hasta 12 horas (Goldsmith et al., 2015; Monte, Zane y Heard, 2015).

Otras formas de consumo con arraigo entre los consumidores de cannabis son: cannabis macerado en alcohol, que consiste básicamente en obtener una tintura de aceite de hachís con alcohol, infusiones de cannabis o bien por vía transdérmica a través de cremas o supositorios (Isorna, Villanueva, Veiga y Otero, 2020; Ramos, 2017).

Debido a su argot como "marihuana sintética" y a la confusión creada en muchos consumidores, cada día es más frecuente en la atención en adicciones encontrarnos con consumidores más jóvenes de cannabinoides sintéticos (también denominados "Spice", "hardcore" o "pescao"). Las personas pueden consumir estas sustancias debido a su fácil adquisición, bajo coste y dificultad en su control toxicológico, pues no se detectan en los controles de cannabis por parte de la policía ni tampoco son detectados en los análisis de orina o sangre ordinarios. Se trata de un preparado con varias hierbas secas embadurnadas con cannabinoides sintéticos, la gran mayoría de la familia de los JWH's (JWH-018, CP-47,497, CP-47,497-C8 y HU-210) que se fuman, aunque en principio no llevan ni tabaco ni marihuana. Estos compuestos comprenden más de 100 sustancias de distintas estructuras químicas que tienen en común su acción sobre el receptor cannabinoide CB1 pero, a diferencia del THC que es un agonista parcial, estas suelen ser agonistas totales y mucho más potentes que el THC, por lo que los efectos psicoactivos son más potentes pero de menor duración por lo que la redosificación y sobredosis son más probables ( $\mathrm{Su}$, Seely, Moran y Hoffman, 2015). Al publicitarse como hierbas naturales, se pretende minimizar la sensación de peligro (Dolengevich-Segal, Gómez-Arnau, Rodríguez-Salgado, Rabito-Alcón y Correas-Lauffer, 2014). Hay otros productos tipo Spice que contienen preparados vegetales, como el Zen, el Skunk o el K2. Se diferencian por el nombre, el etiquetado y el tipo de cannabinoides que contienen. Los nombres y las presentaciones están en constante evolución para evitar el control legal. Su precio oscila entre 9 y 12 euros el gramo, lo que la hace una droga muy económica. Sus efectos psicoactivos son similares a los del cannabis, pero con más efectos adversos dado que pueden actuar sobre otros receptores y se han descrito problemas renales, cardiacos, digestivos y neurológicos no presentes con el consumo de cannabis (Tung, Chiang y Lam, 2012). Hay descrito algún caso de psicosis tóxica esquizofreniforme (Müller et al., 2010) y en foros de usuarios se habla de síntomas psicóticos transitorios; sin embargo, re- cientemente se ha reportado la influencia de esta sustancia en síntomas psicóticos mantenidos (Durand, Delgado, Parra-Pellot y Nichols-Vinueza, 2015; Papanti et al., 2013). Se han descrito reagudización de psicosis, psicosis inducidas y psicosis persistentes con estas sustancias, llegándose a proponer el término "spiceophrenia" (Papanti et al., 2013).

\section{Fumar en cachimba, una nueva moda muy nociva}

Según el OEDT (2020), algo más del 47\% de los estudiantes han fumado tabaco utilizando cachimbas, no apreciándose diferencias significativas en función del sexo. Esta práctica está considerada como una nueva amenaza en la lucha global contra el tabaco y sus consecuencias (Maziak et al., 2015; WHO Study Group on Tobacco Product Regulation, 2015) . De la misma manera, en cuanto al modo de consumo del cannabis, el porro o canuto es la forma más extendida (el 91,4\% del alumnado la han señalado), seguido del uso "pipas de agua" "cachimbas" o "shishas" (10,3\%; chicos: 14,3\%; chicas: 5,8\%) (PNSD, 2021). El aumento del número de adeptos a esta forma de consumo se basa en una serie de mitos como que fumar en cachimba es más sano porque creen que el humo se enfría antes de entrar en los pulmones; que el agua que forma parte del mecanismo filtra las sustancias tóxicas del tabaco; que el humo de la cachimba es menos irritante para la garganta y el tracto respiratorio; que como el tabaco está aromatizado con sabor a frutas en menos dañino, creencias que son falsas. Además del humo del tabaco, los fumadores de cachimba inhalan el humo de las pastillas de carbón que utilizan para encender el tabaco, por lo tanto, están inhalando el gas producto de la combustión del carbón, el cual contiene metales pesados que suponen un riesgo importante para la salud (Pratiti y Mukherjee, 2019) .

Fumar en cachimba es una práctica eminentemente social (varias personas comparten la pipa), esto es, todos aspiran a través de la misma boquilla que pasa de boca en boca, lo que supone una fuente de transmisión de enfermedades infecciosas (Sterling y Mermelstein, 2011). Por otro lado, son cada vez más los adolescentes y jóvenes que mezclan el tabaco con los derivados del cannabis (marihuana y/o hachís) y sustituyen el agua por bebidas alcohólicas, lo cual e indudablemente hacen que esta práctica sea todavía más peligrosa.

Si bien el filtro de agua puede hacer creer a los fumadores que parezca inocuo o menos dañino que fumar cigarrillos o porros directamente, el humo inhalado contiene además de la nicotina y el THC, compuestos tóxicos como el monóxido de carbono, formaldehído, hidrocarburos poliaromáticos, arsénico y plomo (Albisser, Schmidlin, Schindler, Tamm y Stolz, 2013) de esta forma los riesgos se multiplican exponencialmente, pues, además de los conocidos del tabaco y cannabis, se incorporan otros nuevos 
derivados de la combustión del carbón. Según la revisión realizada por Primack et al. (2016) cuando se comparan el consumo de tabaco en una sesión de cachimba frente a un solo cigarrillo; el consumo de tabaco en cachimba suponía inhalar 56 veces más humo, 25 veces más alquitrán; 2,5 veces más nicotina y 10 veces más monóxido de carbono. Traducido en cigarrillos, algunos expertos establecen la equivalencia de entre 25 y 50 cigarrillos para una sola sesión de cachimba (Cobb, Schihadeh, Weaver y Eissenberg, 2011).

Debemos destacar que el consumo de tabaco en cachimba se está extendiendo y afectando, sobre todo, a los más jóvenes. Las actitudes sociales permisivas favorecen su uso entre los y las adolescentes, lo que sin lugar a duda se configura como un factor de iniciación al consumo de cigarrillos y quizás a otras sustancias como el cannabis y sus derivados.

\section{De los hipnosedantes (con o sin receta) al pharming}

En toxicología clínica el "pharming" es el consumo de medicamentos con finalidad "recreativa", generalmente prescritos para un enfermo diferente al usuario, utilizando una dosis distinta a la indicada como terapéutica y buscando alguno de sus efectos psicoactivos (Burillo-Putze et al., 2013). Si bien en Estados Unidos es un fenómeno de gran aceptación por parte de adolescentes y jóvenes, en Europa esta práctica en contextos recreativos y de ocio nocturno va en aumento (United Nations Office on Drugs and Crime, 2011). En España existen pocos datos sobre el fenómeno del "pharming" y comenzó a investigarse a través del consumo de tranquilizantes y somníferos con o sin receta médica en el año 2005 en la encuesta EDADES. Entre 2005 y 2018 se observó un aumento importante de la prevalencia de consumo alguna vez en la vida, pasando de $5,1 \%$ en 2005 a un $19,6 \%$ en 2018 (ESTUDES, 2021), siendo este consumo mayor entre las chicas.

Este consumo es una práctica arraigada entre los jóvenes debido a que es percibida como segura sin la supervisión de un profesional sanitario (Schifano y Chiappini, 2018). Una serie de factores influyen en la creciente popularidad del "pharming": su fácil accesibilidad tanto desde el botiquín del hogar, como mediante su adquisición directa sin receta en farmacias o a través de internet (Burillo-Putze et al., 2013), el hecho de ser sustancias legales y la percepción de que al ser medicamentos son productos seguros y mucho menos peligrosos que las drogas de la calle, así como la falta de percepción de su uso como drogas por parte de los padres u otros familiares o conocidos. Además de la peligrosidad inherente al consumo abusivo propiamente dicho, el riesgo se incrementa por el hecho de utilizarse varios fármacos de forma conjunta, en muchas ocasiones mezclados con alcohol u otras drogas (Burillo-Putze et al., 2012). En este sentido, se ha observado entre jóvenes estadounidenses el uso del denominado "trail mix", consistente en reuniones en las que cada asistente recolecta fármacos del botiquín de su casa, mezclándolos luego en un recipiente, para consumirlos de forma aleatoria (Prosser y Nelson, 2012). Otro aspecto para tener en cuenta con el pharming es la posibilidad de que sirva como puerta de entrada al consumo de otras drogas ilegales, como ocurre con el "cheese", mezcla de heroína con antigripales (fundamentalmente difenhidramina y acetaminofén), que causa euforia y alucinaciones tras inhalarlo y que es conocida como la "heroína de inicio" (Maxwell, Coleman, Feng, Goto y Tirado, 2012). También se pueden encontrar fácilmente en internet recursos y sugerencias de posibles combinaciones de sustancias para aumentar el efecto eufórico de la loperamida (por ejemplo, http://www.bluelight.ru; http://www.drugs-forum.com).

La Agencia Europea de Medicamento (Schifano y Chiappini, 2018) ha observado un aumento en los últimos años tanto en la prescripción como en la disponibilidad de antipsicóticos de segunda generación (SGA) como la quetiapina. Otro medicamento utilizado es la loperamida que utilizada a bajas dosis es un potente agonista del receptor $m u$-opioide y consumida en grandes cantidades (más de 50mgr) produce efectos eufóricos, depresión del sistema nervioso central posiblemente mejor euforia inicial seguida de depresión del SNC y cardiotoxicidad (Eggleston, Clark y Marraffa, 2017; Schifano y Chiappini, 2018), también se la denomina "metadona del pobre" (Stanciu y Gnanasegaram, 2017).

Finalmente, en relación con el fenómeno del "pharming”, no solo hay que pensar en su consumo en espacios de ocio o recreativos, sino que podríamos considerar como tal el uso de medicamentos para dormir o relajarse sin ánimo lúdico o también para no dormir, en aquellas personas que tienen que trabajar de noche o estudiar en tiempos de examen o para combatir el efecto de medicamentos o drogas que les impidan conciliar el sueño (Alfaro y Hernández, 2019; Bennett y Holloway, 2017).

\section{Conclusiones}

Los datos que presentan el PNSD y el OEDT revelan la importancia del consumo de drogas en la sociedad actual y reflejan un cambio tanto en las sustancias mismas como en los patrones de consumo e incluso en el perfil epidemiológico de los consumidores, lo cual conlleva la necesidad de actualizar los conocimientos sobre el abordaje de las posibles complicaciones médicas y psicopatológicas. Nos encontramos con un panorama novedoso, cambiante y con escasos datos empíricos, lo que supone un reto para los profesionales en el momento de abordar estas nuevas formas de consumo de drogas y sus consecuencias. Nuevas sustancias y formas hasta ahora desconocidas en el acceso, la venta y la distribución de drogas ponen en cuestión los métodos de vigilancia, detección y prevención actuales. El anonimato que aporta internet, la sencillez para la compra, 
los bajos precios y, en ocasiones, la ausencia de un marco legal expande su consumo entre los más jóvenes.

La utilización de drogas y de las nuevas formas de consumo es un problema de gran envergadura, que afecta a la vida personal, laboral y familiar de muchas personas y que está generando otro consumo que, hasta hace poco, era escasamente valorado: el de los recursos del sistema sanitario. Tal y como sugiere Nogué, Amigó y Galicia (2014), el consumo de sustancias ha dejado de ser un problema individual y es, de pleno derecho, algo que afecta a toda la sociedad. Los consumidores de drogas deberían saber que su destino final puede ser un servicio de urgencias y, en otras ocasiones, un Instituto Médico-Legal.

\section{Conflicto de intereses}

Los autores declaran no tener ningún conflicto de intereses y que no han recibido ningún tipo de financiación para la realización de este artículo.

\section{Referencias}

Adán, C. (2018). Pulseras de colores para controlar que no se venda alcohol a menores. Heraldo. Recuperado de https://www.heraldo.es/noticias/aragon/2016/12/31/ pulseras-colores-para-controlar-que-venda-alcohol-menores-los-cotillones-1151079-300.html.

Agencia Española del Medicamento y Productos Sanitarios (2016). Boletín mensual de la AEMPS sobre medicamentos de uso humano. Septiembre de 2016. Recuperado de https://www.aemps.gob.es/informa/ boletines-AEMPS/boletinMensual/2016/septiembre/ boletin-septiembre.htm.

Agnich, L. E., Stogner, J. M., Miller, B. L. y Marcum, C. D. (2013). Purple drank prevalence and characteristics of misusers of codeine cough syrup mixtures. Addictive Behaviors, 38, 2445-2449. doi:10.1016/j. addbeh.2013.03.020.

Albisser, S., Schmidlin, J., Schindler, C., Tamm, M. y Stolz, D. (2013). Water pipe smoking and its association with cigarette and cannabis use in young adults in Switzerland. Respiration, 86, 210-215. doi:10.1159/000342894.

Alfaro, F. A. y Hernández, N. (2019). Uso recreativo de benzodiacepinas en la población joven. Revista Ene De Enfermería, 13, 1-19.

Anderson, R. P. y Zechar, K. (2019). Lung injury from inhaling butane hash oil mimics pneumonia. Respiratory Medicine Case Reports, 26, 171-173. doi:10.1016/j. rmcr.2019.01.002.

Arria, A., Caldeira, K., Kasperski, S. J., Vincent, K. B., Griffiths, R. R. y O’Grady, K. E. (2011). Energy drink consumption and increased risk for alcohol dependence. Alcoholism: Clinical and Experimental Research, 35, 365-375. doi:10.1111/j.1530-0277.2010.01352.x.
Báez-Ferrer, N., Parra-Esquivel, P. C., Gálvez-Rodríguez, M., Burillo-Putze, G., Abreu-González, P. y DomínguezRodríguez, A. (2020). Miocarditis relacionada con el uso de bebidas energéticas: A propósito de un caso. Emergencias, 32.

Bennett, T. y Holloway, K. (2017). Motives for illicit prescription drug use among university students: A systematic review and meta-analysis. International Journal of Drug Policy, 44, 12-22. doi:10.1016/j.drugpo.2017.02.012.

Bersani, S., Corazza, O., Albano, G., Bruschi, S., Minichino, A., Vicinanza, R.,... Schifano, F. (2015). The "Eyeballing" technique: An emerging and alerting trend of alcohol misuse. European Review for Medical and Pharmacological Sciences, 19, 2311-2317.

Blakley, B. W. y Schilling, H. (2008). Deafness associated with acetaminophen and codeine abuse. Journal of Otolaryngology-Head E Neck Surgery, 37.

Brown, A. K., Nagelhout, G. E., van den Putte, B., Willemsen, M. C., Mons, U., Guignard, R. y Thompson, M. E. (2015). Trends and socioeconomic differences in rollyour-own tobacco use: Findings from the ITC Europe Surveys. Tobacco Control, 24 (Supl. 3), 11-16. doi:10.1136/ tobaccocontrol-2014-051986.

Budney, A. J., Sargent, J. D. y Lee, D. C. (2015). Vaping cannabis (marijuana): Parallel concerns to e-cigs? Addiction, 110, 1699-1704. doi:10.1111/add.13036.

Buglass, A. J. (2011). Handbook of alcoholic beverages: Technical, analytical and nutritional aspects. John Wiley \& Sons Ltd.

Burillo-Putze, G., Aldea-Perona, A., Rodríguez-Jiménez, C., García-Saiz, M., Climent, B., Dueñas, A.,... Hoffman, R. S. (2013). Drogas emergentes (II): El pharming. Anales Del Sistema Sanitario De Navarra, 36, 99-114. doi:10.4321/ S1137-66272013000100010.

Burillo-Putze, G., Hernández, M.J. y Echeverría, P. (2012). New ways of consuming alcohol. Anales de Pediatría, 77, 419-420. doi:10.1016/j.anpedi.2012.03.017.

Calduch, T. C., Jiménez, C. H., San Segundo, T., Valle, M. y Carlos-Roca, A. P. (2012). Tabaco de liar: Una prioridad de salud pública y consumo. Gaceta Sanitaria, 26, 267-269. doi:10.1016/j.gaceta.2011.09.010.

Caudevilla, F. (2015). Pedro, el Lobo y las drogas de verano. Pediatría Atención Primaria, 17, 203-204. doi:10.4321/ S1139-76322015000400001.

Chambers, R. A. (2008). Drunkorexia. Journal of Dual Diagnosis, 4, 414-416. doi:10.1080/15504260802086677.

Chiappini, S., Schifano, F., Corkery, J. M.y Guirguis, A. (2021). Beyond the 'purple drank': Study of promethazine abuse according to the European Medicines Agency adverse drug reaction reports. Journal of Psychopharmacology, 35, 681-692. doi:10.1177/0269881120959615.

Cobb, C. O., Shihadeh, A., Weaver, M. F. y Eissenberg, T. (2011). Waterpipe tobacco smoking and cigarette smoking: A direct comparison of toxicant exposure and 
subjective effects. Nicotine E Tobacco Research, 13, 78-87. doi:10.1093/ntr/ntq212.

Córdoba, R. (2014). El desafío de los cigarrillos electrónicos. Atención Primaria, 46, 307-312. doi:10.1016/j. aprim.2014.01.002.

Di Forti, M., Quattrone, D., Freeman, T. P., Tripoli, G., Gayer-Anderson, C., Quigley, H.,... La Cascia, C. (2019). The contribution of cannabis use to variation in the incidence of psychotic disorder across Europe (EU-GEI): A multicentre case-control study. The Lancet Psychiatry, 6, 427-436. doi:10.1016/S2215-0366(19)30048-3.

Dolengevich-Segal, H., Gómez-Arnau, J., Rodríguez-Salgado, B., Rabito-Alcón, M. F. y Correas-Lauffer, J. (2014). Panorama actual en el uso de drogas emergentes. Salud y Drogas, 14, 47-58.

Durand, D., Delgado, L. L., Parra-Pellot, D. M. de la. y NicholsVinueza, D. (2015). Psychosis and severe rhabdomyolysis associated with synthetic cannabinoid use: A case report. Clinical Schizophrenia E Related Psychoses, 8, 205-208.

Eggleston, W., Clark, K. H. y Marraffa, J. M. (2017). Loperamide abuse associated with cardiac dysrhythmia and death. Annals of Emergency Medicine, 69, 83-86. doi:10.1016/j.annemergmed.2016.03.047.

El Dib, R., Suzumura, E. A., Akl, E. A., Gomaa, H., Agarwal, A., Chang, Y.,... Maziak, W. (2017). Electronic nicotine delivery systems and/or electronic non-nicotine delivery systems for tobacco smoking cessation or reduction: A systematic review and meta-analysis. BMJ Open, 7, e012680. doi:10.1136/bmjopen-2016-012680.

Elwood, W. N. (2001). Sticky business: Patterns of procurement and misuse of prescription cough syrup in Houston. Journal of Psychoactive Drugs, 33, 121-133. doi:10. 1080/02791072.2001.10400477.

Encuesta Europea sobre Alcohol y otras Drogas (2019). Informe ESPAD 2019. Recuperado de https://pnsd. sanidad.gob.es/profesionales/sistemasInformacion/ sistemaInformacion/pdf/20201112_Informe_ ESPAD_2019_final.pdf.

Fonseca, L. (2013). Detectados cuatro casos en Asturias de chicas intoxicadas por tampones con vodka. El Comercio. Recuperado de https://www.elcomercio.es/v/20130521/ gijon/gijon-registro-tres-casos-20130521.html.

French, C. E., Coope, C. M., McGuinness, L. A., Beck, C. R., Newitt, S., Ahyow, L.,... Oliver, I. (2019). Cannabis use and the risk of tuberculosis: A systematic review. BMC Public Health, 19, 1-13. doi:10.1186/s12889-019-7127-0.

Goldsmith, R. S., Targino, M. C., Fanciullo, G. J., Martin, D. W., Hartenbaum, N. P., White, J. M. y Franklin, P. (2015). Medical marijuana in the workplace: Challenges and management options for occupational physicians. Journal of Occupational and Environmental Medicine, 57, 518. doi:10.1097/JOM.0000000000000454.
Gotts, J. E., Jordt, S. E., McConnell, R. y Tarran, R. (2019). What are the respiratory effects of e-cigarettes? BMJ, 366 doi:10.1136/bmj.15275.

Hancock-Allen, J. B., Barker, L., VanDyke, M. y Holmes, D. B. (2015). Death following ingestion of an edible marijuana product-Colorado, March 2014. MMWR. Morbidity and Mortality Weekly Report, 64, 771. doi:10.15585/mmwr. mm6428a6.

Hart, M., Agnich, L. E., Stogner, J. y Miller, B. L. (2014). 'Me and my drank': Exploring the relationship between musical preferences and purple drank experimentation. American Journal of Criminal Justice, 39, 172-186. doi:10.1007/s12103-013-9213-7.

Hazekamp, A., Ware, M. A., Muller-Vahl, K. R., Abrams, D. y Grotenhermen, F. (2013). The medicinal use of cannabis and cannabinoids - an international cross-sectional survey on administration forms. Journal of Psychoactive Drugs, 45, 199-210. doi:10.1080/02791072.2013.805976.

Isorna, M., Villanueva, V., Veiga, S. y Otero, M. (2020). Formas de consumo del cannabis: Características, riesgos y daños asociados. En M. Isorna, A. Rial y V. Villanueva (Eds.), Cannabis: Evidencia científica vs controversia social (pp. 59-101). Dykinson, S.L.

Izquierdo Fos, I., Vázquez Gomis, R. M., Vázquez Gomis, C., Piernas, R., Climent Forner, E.,... Vargas Torcal, F. (2012). Episodio de fibrilación auricular tras ingesta de bebida energética y práctica de deporte. Anales de Pediatría, 77, 417-419. doi:10.1016/j.anpedi.2012.05.006.

Jackler, R. K. y Ramamurthi, D. (2019). Nicotine arms race: JUUL and the high-nicotine product market. Tobacco Control, 28, 623-628. doi:10.1136/ tobaccocontrol-2018-054796.

Joseph, S., Krebs, N. M., Zhu, J., Wert, Y., Goel, R., Reilly, S. M.,... Cheriyath, P. (2018). Differences in nicotine dependence, smoke exposure and consumer characteristics between smokers of machine-injected roll-your-own cigarettes and factory-made cigarettes. Drug and Alcohol Dependence, 187, 109-115. doi:10.1016/j. drugalcdep.2018.01.039.

Kershaw, S. (2008). Starving themselves, cocktail in hand. The New York Times. Recuperado de https:/ /www.nytimes. com/2008/03/02/fashion/02drunk.html.

Laugesen, M., Epton, M., Frampton, C. M., Glover, M. y Lea, R. A. (2009). Hand-rolled cigarette smoking patterns compared with factory-made cigarette smoking in New Zealand men. BMCPublic Health, 9, 1-6. doi:10.1186/14712458-9-194.

Lessenger,J. E. y Feinberg, S. D. (2008). Abuse of prescription and over-the-counter medications. The Journal of the American Board of Family Medicine, 21, 45-54. doi:10.3122/ jabfm.2008.01.070071.

Manzoni, C. (2014). Más y más litros: Las bebidas alcohólicas "listas para tomar" ya son furor en el país. $L a$ Nación. Recuperado de https://www.lanacion.com.ar/ 
economia/mas-y-mas-litros-las-bebidas-alcoholicas-listaspara-tomar-ya-son-furor-en-el-pais-nid1728936/.

Marconi, A., Di Forti, M., Lewis, C. M., Murray, R. M. y Vassos, E. (2016). Meta-analysis of the association between the level of cannabis use and risk of psychosis. Schizophrenia Bulletin, 42, 1262-1269. doi:10.1093/schbul/sbw003.

Martínez, A. G., López-Espinoza, A., Navarro Meza, M., López-Uriarte, P. y Salazar Estrada, J. G. (2014). Trastornos de la conducta de beber: Una propuesta de investigación. Revista Mexicana De Trastornos Alimentarios, 5, 58-69.

Maxwell, J. C., Coleman, J. J., Feng, S., Goto, C. S. y Tirado, C. F. (2012). Cheese: An old drug in a new wrapper. Drug and Alcohol Dependence, 126, 161-167. doi:10.1016/j. drugalcdep.2012.05.015.

Maziak, W., Taleb, Z. B., Bahelah, R., Islam, F., Jaber, R., Auf, R. y Salloum, R. G. (2015). The global epidemiology of waterpipe smoking. Tobacco Control, 24 (Supl. 1), 3-12. doi:10.1136/tobaccocontrol-2014-051903.

Miuli, A., Stigliano, G., Lalli, A., Coladonato, M., D’Angelo, L., Esposito, F.,... Schifano, F. (2020). "Purple drank" (codeine and promethazine cough syrup): A systematic review of a social phenomenon with medical implications. Journal of Psychoactive Drugs, 52, 453-462. doi:10.1080/027 91072.2020.1797250.

Monraz-Pérez, S., Regalado-Pineda, J. y Pérez-Padilla, R. (2015). El cigarrillo electrónico: Peligro u oportunidad. Neumología Y Cirugía De Tórax, 74, 82-86.

Monte, A. A., Zane, R. D. y Heard, K. J. (2015). The implications of marijuana legalization in Colorado. Jama, 313, 241-242. doi:10.1001/jama.2014.17057.

Müller, H., Sperling, W., Köhrmann, M., Huttner, H. B., Kornhuber,J.y Maler,J. (2010). The synthetic cannabinoid Spice as a trigger for an acute exacerbation of cannabis induced recurrent psychotic episodes. Schizophrenia Research, 118, 309-310. doi:10.1016/j.schres.2009.12.001.

Nogué, S., Amigó, M. y Galicia, M. (2014). Raves, consumo de drogas y asistencia en urgencias. Adicciones, 26, 189190.

O’Brien, M. C., McCoy, T. P., Rhodes, S. D., Wagoner, A. y Wolfson, M. (2008). Caffeinated cocktails: Energy drink consumption, high-risk drinking, and alcoholrelated consequences among college students. Academic Emergency Medicine, 15, 453-460. doi:10.1111/j.15532712.2008.00085.x.

Observatorio Español de Drogodependencias y toxicomanías (2020). Encuesta sobre el uso de drogas en enseñanzas secundarias en España (ESTUDES). Madrid: Ministerio de Sanidad. Delegación del gobierno para el plan nacional sobre drogas. Recuperado de https://pnsd.sanidad.gob. es/profesionales/sistemasInformacion/sistemaInformacion/pdf/ESTUDES_2020_Informe.pdf.

Observatorio Europeo de las Drogas y las Toxicomanías (2019). Informe Europeo sobre Drogas. Tendencias y novedades 2019. Lisboa: Observatorio Europeo de las Drogas y las Toxicomanías. Recuperado de https://www.emcdda. europa.eu/system/files/publications/11364/20191724_ TDAT19001ESN_PDF.pdf.

Oeltmann, J. E., Oren, E., Haddad, M. B., Lake, L. K., Harrington, T. A., Ijaz, K. y Narita, M. (2006). Tuberculosis outbreak in marijuana users, Seattle, Washington, 2004. Emerging Infectious Diseases, 12, 1156. doi:10.3201/ eid1207.051436.

Orr, C., Spechler, P., Cao, Z., Albaugh, M., Chaarani, B., Mackey, S.,... Bokde, A. L. (2019). Grey matter volume differences associated with extremely low levels of cannabis use in adolescence. Journal of Neuroscience, 39, 1817-1827. doi:10.1523/JNEUROSCI.3375-17.2018.

Oteri, A., Salvo, F., Caputi, A. P. y Calapai, G. (2007). Intake of energy drinks in association with alcoholic beverages in a cohort of students of the School of Medicine of the University of Messina. Alcoholism: Clinical and Experimental Research, 31, 1677-1680. doi:10.1111 /j.1530-0277.2007.00464.

Papanti, D., Schifano, F., Botteon, G., Bertossi, F., Mannix, J., Vidoni, D.,... Bonavigo, T. (2013). "Spiceophrenia”: A systematic overview of "Spice"-related psychopathological issues and a case report. Human Psychopharmacology: Clinical and Experimental, 28, 379-389. doi:10.1002/ hup.2312.

Pascual, F. (2002). Percepción del alcohol entre los jóvenes. Adicciones, 14, 123-131. doi:10.20882/adicciones.522.

Perlman, D. C., Perkins, M. P., Paone, D., Kochems, L., Salomon, N., Friedmann, P. y Des Jarlais, D. C. (1997). "Shotgunning" as an illicit drug smoking practice. Journal of Substance Abuse Treatment, 14, 3-9. doi:10.1016/S07405472(96)00182-1.

Pietrabissa, G., Rossi, A., Gaudenzi, M., Bertuzzi, V., Tagliagambe, A., Volpi, C.,... Castelnuovo, G. (2018). Drunkorexia: Empirical investigation and analysis of the characteristics of the phenomenon in an Italian sample of adolescents and young adults. Psychology, Society, E Education, 10, 285-299. doi:10.25115/psye.v10i3.2135.

Plan Nacional sobre Drogas. (2021). ESTUDES 2021. Encuesta sobre uso de drogas en enseñanzas secundarias en España, 1994-2021. Madrid: Ministerio de Sanidad. Recuperado de https://pnsd.sanidad.gob.es/profesionales/ sistemasInformacion/sistemaInformacion/pdf/ESTUDES_2021_Informe_de_Resultados.pdf.

Pratiti, R. y Mukherjee, D. (2019). Epidemiology and adverse consequences of hookah/waterpipe use: A systematic review. Cardiovascular $\mathcal{E}$ Hematological Agents in Medicinal Chemistry (Formerly Current Medicinal ChemistryCardiovascular E゚ Hematological Agents), 17, 82-93. doi:10.2 174/1871525717666190904151856.

Primack, B. A., Carroll, M. V., Weiss, P. M., Shihadeh, A. L., Shensa, A., Farley, S. T.,.. Nayak, S. (2016). Systematic review and meta-analysis of inhaled toxicants from 
waterpipe and cigarette smoking. Public Health Reports, $131,76-85$.

Prosser, J. M. y Nelson, L. S. (2012). The toxicology of bath salts: A review of synthetic cathinones. Journal of Medical Toxicology, 8, 33-42. doi:10.1007/s13181-011-0193-z.

Raber, J. C., Elzinga, S. y Kaplan, C. (2015). Understanding dabs: Contamination concerns of cannabis concentrates and cannabinoid transfer during the act of dabbing. The Journal of Toxicological Sciences, 40, 797-803. doi:10.2131/ jts.40.797.

Ramos, J. (2017). Efectos terapéuticos de los cannabinoides. Instituto universitario de Investigación en Neuroquímica de la Universidad Complutense de Madrid.

Rehm, J., Shield, K. D., Joharchi, N. y Shuper, P. A. (2012). Alcohol consumption and the intention to engage in unprotected sex: Systematic review and meta-analysis of experimental studies. Addiction, 107, 51-59. doi:10.1111/ j.1360-0443.2011.03621.x.

Rial, A., Burkhart, G., Isorna, M., Barreiro, C., Varela, J. y Golpe, S. (2018). Consumo de cannabis entre adolescentes: Patrón de riesgo, implicaciones y posibles variables explicativas. Adicciones, 31, 64-77. doi:10.20882/ adicciones.1212.

Rial, A., Golpe, S., Barreiro, C., Gómez, P. e Isorna, M. (2020). La edad de inicio en el consumo de alcohol en adolescentes: Implicaciones y variables asociadas. Adicciones, 32, 52-62. doi:10.20882/adicciones.1266.

Rolke, H. B., Bakke, P. S. y Gallefoss, F. (2009). Relationships between hand-rolled cigarettes and primary lung cancer: A Norwegian experience. The Clinical Respiratory Journal, 3, 152-160. doi:10.1111/j.1752-699X.2008.00125.x.

Saenz, H. (2011). Baleares pone fin a los "chupitos de la risa”. El Mundo. Recuperado de https://www.elmundo. es/elmundo/2011/08/24/baleares/1314195899.html.

Schifano, F. y Chiappini, S. (2018). Is there such a thing as a'lope'dope? Analysis of loperamide-related European Medicines Agency (EMA) pharmacovigilance database reports. PLoS One, 13. doi:10.1371/journal.pone.0204443.

Signes-Costa, J., de Granda-Orive, J. I., Pinedo, Á R., Escrig, A. C., de Higes Martínez, E.,... Jiménez-Ruiz, C. A. (2019). Declaración Oficial de la Sociedad Española de Neumología y Cirugía Torácica (SEPAR) sobre cigarrillos electrónicos e IQOS ${ }^{\circledR}$. Archivos De Bronconeumología, 55, 581-586. doi:10.1016/j.arbres.2019.04.023.

Stanciu, C. N. y Gnanasegaram, S. A. (2017). Loperamide, the "poor man's methadone": Brief review. Journal of Psychoactive Drugs, 49, 18-21. doi:10.1080/02791072.2016 .1260188 .

Stephens, D., Patel, J. K., Angelo, D. y Frunzi, J. (2020). Cannabis butane hash oil dabbing induced lung injury mimicking atypical pneumonia. Cureus, 12. doi:10.7759/ cureus.7033.

Sterling, K. L. y Mermelstein, R. (2011). Examining hookah smoking among a cohort of adolescent ever smokers.
Nicotine EF Tobacco Research, 13, 1202-1209. doi:10.1093/ $\mathrm{ntr} / \mathrm{ntr} 146$.

Stogner, J. M. y Miller, B. L. (2015). The dabbing dilemma: A call for research on butane hash oil and other alternate forms of cannabis use. Substance Abuse, 36, 393-395. doi:1 0.1080/08897077.2015.1071724.

Su, M. K., Seely, K. A., Moran, J. H. y Hoffman, R. S. (2015). Metabolism of classical cannabinoids and the synthetic cannabinoid JWH-018. Clinical Pharmacology $\mathcal{E}$ Therapeutics, 97, 562-564. doi:10.1002/cpt.114.

Torres, F. (2020). Analizamos el estado de salud de los festivales españoles. Recuperado de https://wololosound.com/ articulos/informe-festivales-espanoles/.

Tung, C. K., Chiang, T. P. y Lam, M. (2012). Acute mental disturbance caused by synthetic cannabinoid: A potential emerging substance of abuse in Hong Kong. East Asian Archives of Psychiatry, 22, 31-33. doi:10.3316/ informit.870000302755965.

United Nations Office on Drugs and Crime (2011). The non-medical use of prescription drugs. Policy direction issues. Viena: United Nations. Recuperado de https://www. unodc.org/documents/drug-prevention-and-treatment/ nonmedical-use-prescription-drugs.pdf.

Upev (2019). El cigarrillo electrónico duplica su negocio en España. Recuperado de https://upev.org/resumen-2019junio-semana-4/.

Villarino, A. (2012). Trastornos de la conducta alimentaria. No todo es anorexia y bulimia. Controversias Sobre Los Trastornos Alimentarios, 33-46.

Volkow, N. D., Swanson, J. M., Evins, A. E., DeLisi, L. E., Meier, M. H., González, R.,... Baler, R. (2016). Effects of cannabis use on human behavior, including cognition, motivation, and psychosis: A review. JAMA Psychiatry, 73, 292-297. doi:10.1073/pnas.1411228111.

Welsh, C., Goldberg, R., Tapscott, S., Medoff, D., Rosenberg, S. y Dixon, L. (2012). "Shotgunning" in a population of patients with severe mental illness and comorbid substance use disorders. The American Journal on Addictions, 21, 120-125. doi:10.1111/j.1521-0391.2011.00201.x.

WHO. Study Group on Tobacco Product Regulation (2015). Advisory note: Waterpipe tobacco smoking: Health effects, research needs and recommended actions for regulators. Recuperado de https://apps.who.int/iris/ bitstream/handle/10665/161991/9789241508469_eng. pdf.

World Health Organization (2016). Health and Social Effects of Nonmedical Cannabis Use (The). World Health Organization. Recuperado de https://www.who.int/ publications/i/item/9789241510240.

Young, D., Yong, H., Borland, R., Shahab, L., Hammond, D., Cummings, K. M. y Wilson, N. (2012). Trends in rollyour-own smoking: Findings from the ITC Four-Country Survey (2002-2008). Journal of Environmental and Public Health, 2012. doi:10.1155/2012/406283. 